\title{
Characterization of the Atmospheric Depth Profile using the ground level temperature: the case of Malargüe, Argentina
}

\author{
Juan Cruz MORENO ${ }^{1,2}$ and Sergio SCIUTTO ${ }^{1,2}$ \\ ${ }^{1}$ Departamento de Física. Facultad de Ciencias Exactas. Universidad \\ Nacional de La Plata. Calles 49 y 115, La Plata, Argentina. \\ ${ }^{2}$ Instituto de Física La Plata. Consejo Nacional de Investigaciones \\ Cientificas y Tecnológicas (CONICET). La Plata, Argentina
}

\begin{abstract}
We present a study of the atmospheric depth profile and the dependence with its characteristic parameters. We introduce a new model, named GAMMA, based on a parameterization that allows us to obtain the atmospheric depth profile specifying only one simple physical parameter, namely the temperature at ground. The GAMMA Model consists of a multilayer representation of the atmosphere that can be adjusted conveniently via constrained fits that are built to ensure interlayer continuity for both atmospheric depth and density profiles. Our analysis uses experimental data collected at Malargüe, Argentina by meteorological radiosondes. The GAMMA Model can reproduce the averaged atmospheric depth profiles in all the cases available for analysis with good accuracy. The relative differences between model predictions and averaged data are always less than approximately $0.7 \%$.
\end{abstract}

\section{INTRODUCTION}

The accurate characterization of the atmosphere constitutes a critical task for the analysis of extensive air showers generated by cosmic rays. The list of cosmic ray experimental collaborations that make major efforts in atmospheric monitoring is extensive. Experiments like Pierre Auger Observatory, HiRes, HESS, Veritas, AGASA, etc., have documented their work on this field ([1], [2], [3], [4], [5]).

A particular important quantity to study is the vertical atmospheric depth, $X(h)$, defined as

$$
X(h)=\int_{h}^{\infty} \rho(\zeta) d \zeta
$$

where $h$ is the altitude, $\rho(h)$ is the matter density, and the integral in (1) is evaluated along a vertical straight line.

For both experimental and simulated air shower data analysis, a precise knowledge of $X(h)$ is essential for an accurate estimation of observable air shower quantities [6-8]. For example, in the fluorescence detection technique the measurements are expressed in terms of geometrical altitude and must be converted into atmospheric depth to obtain the traversed air mass. The opposite case 
can be found in simulations where traversed matter is expressed in terms of the atmospheric depth. To compare it with the experimental measurements a conversion to altitude is required.

It is well known that the Earth's atmosphere is permanently changing, and such changes affect also the vertical depth profile. The most relevant variations observed occur at relatively low altitudes (in the first kilometers above sea level), and are correlated noticeably with the daily and seasonal cycles. Any model capable of describing adequately the profile must take into account such variations. Due to the complexity of the atmospheric dynamics, it is not straightforward to build a model that could reproduce the proper profile with a minimal set of input parameters.

The model we introduce in this article was conceived upon the idea of providing a simple but effective way of reproducing the atmospheric profile under given conditions. Its main characteristic is that the ground level temperature is used as a parameter to efficiently parameterize the different profiles. In the analyzed cases (see below), we found that such parameterization can reproduce the atmospheric profile experimental data with excellent accuracy. Due to its practicality, this model can be easily used in shower reconstruction and shower simulation studies.

This work is organized as follows: section II is an overview of the classical models used to describe the atmospheric depth profile; in section III, we present the new model; section IV is dedicated to the discussion of several important points related to the model; and a summary of this work and our conclusions are placed in section IV. We have also included an appendix summarizing the expressions and the values of the obtained parameterization.

\section{THE ATMOSPHERIC DEPTH PROFILE MODELS}

The mass density of air as a function of altitude is one of the basic observables taken into account by every atmospheric profile model virtually. The density of air diminishes six orders of magnitude when the altitude goes from sea level up to $100 \mathrm{~km}$, and another six orders in the range of $100 \mathrm{~km}$ to $300 \mathrm{~km}$. The chemical composition, usually described by the mean molar mass $\left(M_{m}\right)$, is another relevant parameter to consider. In dry air $M_{m}$ has a constant value of $M_{m}=28.966 \mathrm{~g}$ $\mathrm{mol}^{-1}$ in the range of $0 \leq h \leq 90 \mathrm{~km}$ and begins to decrease monotonically to higher altitudes. The constant value of $M_{m}$ represents the mixing of $78.084 \% \mathrm{~N}, 20.946 \% \mathrm{O}, 0.934 \% \mathrm{Ar}$ and approximately $0.036 \%$ by others elements.

During the last century, many studies and models were developed to characterize the terrestrial atmosphere. A simple model to describe the density variation with altitude, including the molecular weight is the well-known isothermal model, where the air is considered as an ideal gas at a given 
uniform temperature T. A straightforward calculation leads to the following expression for the vertical depth as a function of altitude:

$$
X(h)=X_{0} \quad e^{-g M_{m} h / R T}
$$

where $R$ is the universal gas constant, $g$ is the local gravity acceleration, $M_{m}$ is the molar mass of air, and $X_{0}$ is the vertical atmospheric depth at sea level. In most cases, $T$ is taken as the sea level temperature. $X_{0}$ can be calculated from the density at sea level $\rho_{0}$ via $X_{0}=\rho_{0} R T / g M_{m}$ (notice that from equation (1) it follows that $\rho(h)=-d X(h) / d h)$.

Another widely used and accepted atmospheric model is the so-called US Standard Atmosphere (USStdA 1976) [9], consisting of an idealized atmosphere model based on measurements made at different mid-latitude geographical locations. The USStdA 1976 tables are built from adequate averages of those measurements, and thus this model can be considered to represent a mean global atmosphere.

In the USStdA 1976 model, the atmosphere is represented as a series of consecutive layers covering altitudes from sea level up to $1,000 \mathrm{~km}$. An exhaustive description of the variation with altitude of pressure, temperature, density, and chemical composition, among other quantities, is presented at each layer. One characteristic of the atmosphere that can be clearly appreciated from the USStdA 1976 model is that its temperature varies with altitude [9]. Thus, it is expected that the isothermal model is inappropriate for an accurate description of the atmospheric depth profile in the entire altitude range of interest for this work, from sea level up to about $100 \mathrm{~km}$. However, comparing the isothermal and the USStdA 1976 models, an acceptable agreement of the lowest kilometers is found, but a significant difference appears at altitudes higher than $10 \mathrm{~km}$.

On the basis of these models, J. Linsley developed a parameterization of the atmospheric depth profile [10], that reproduces accurately the average density profile given by the USStdA 1976 model.

The hallmark of the Linsley model is to suppose that the atmosphere can be considered as a series of consecutive layers with fixed boundaries, each of them with a characteristic temperature; and consequently the model is named isothermal by layers. Therefore, and taking into account equation (2), the vertical atmospheric depth can be approximated at each isothermal layer in terms of a function of the form

$$
X(h)=a+b e^{-\frac{h}{c}}
$$

where $a, b$, and $c$ are given parameters. In a series of consecutive layers, those parameters are adjusted to ensure continuity of $X$ as a function of $h$. In the Linsley model, the profile is completed 


\begin{tabular}{|c|cc|ccc|}
\hline $\begin{array}{c}\text { Layer } \\
l\end{array}$ & $\begin{array}{c}\text { Layer } \\
h_{l-1}[\mathrm{~km}]\end{array}$ & $\begin{array}{c}\text { limits } \\
h_{l}[\mathrm{~km}]\end{array}$ & $\begin{array}{c}a_{l} \\
{\left[\mathrm{~g} \mathrm{~cm}^{-2}\right]}\end{array}$ & $\begin{array}{c}b_{l} \\
{\left[\mathrm{~g} \mathrm{~cm}^{-2}\right]}\end{array}$ & $\begin{array}{c}c_{l} \\
{[\mathrm{~m}]}\end{array}$ \\
\hline 1 & 0 & 4 & -186.5562 & 1222.6562 & 9941.8638 \\
2 & 4 & 10 & -94.9199 & 1144.9069 & 8781.5355 \\
3 & 10 & 40 & 0.61289 & 1305.5948 & 6361.4304 \\
4 & 40 & 100 & 0.0 & 540.1778 & 7721.7016 \\
5 & 100 & 112.8 & 0.01128292 & 1.0 & 10000000 \\
\hline
\end{tabular}

TABLE I: Values of the parameterization of Linsley with 5 layers.

with a constant density layer that ends at a maximum finite altitude $h_{\max }$, on top of the previous isothermal layers.

In this way, in a $L-1$ isothermal layer model the complete profile will read as

$$
X(h)=\left\{\begin{array}{ccrl}
a_{l}+b_{l} e^{-\frac{h}{c_{l}}} & h_{l-1} & \leq h<h_{l} \\
a_{L}-b_{L} \frac{h}{c_{L}} & h_{L-1} & \leq h<h_{L} \\
0 & h & \geq h_{L}
\end{array}\right.
$$

where $a_{l}, b_{l}, c_{l}, l=1, \ldots, L$ are given parameters, and the altitudes $h_{0}, \ldots, h_{L}$ define the layer boundaries, that must verify that $h_{l}<h_{l+1}$ for all $l$. Layer $L$, the uppermost one, corresponds to the already mentioned constant density layer that allows to reach a finite maximum altitude $h_{\max }=h_{L}$. Note that $X(h)$ as defined in equation (4), can be inverted analytically to obtain the function $h(X)$ frequently used in air shower simulation algorithms.

Using equation (4) with $L=5$ layers, Linsley parameterized the values of the USStdA 1976 and obtained the values listed in table I, that completely define the widely used Linsley Model of 5 layers. The Linsley profile $X(h)$ is a very accurate parameterization of the USStdA 1976 profile, covering the range that goes from sea level up to $h_{\max } \simeq 112.8 \mathrm{~km}$, altitude that marks the end of the profile with $X\left(h_{\max }\right)=0 \mathrm{~g} \mathrm{~cm}^{-2}$. It is worthwhile mentioning that for altitudes greater than 90 $\mathrm{km}$, the Linsley profile is less accurate than at lower altitudes. Nevertheless, at such altitudes the vertical depth is very small (around $0.001 \mathrm{~g} \mathrm{~cm}^{-2}$ or less) not affecting significantly any air shower calculation. Although it only describes the behavior of the parameter $X$, Linsley parameterization is usually mentioned as the Linsley Model.

Being a parameterization of a global average atmosphere, the Linsley Model is applicable at any geographical location, and at any moment of the year. However, it is a fact that there are obvious changes in the atmospheric depth related with seasonal and local variations in temperature, 
pressure and humidity [11]. The lack of consideration of those changes has been the main motivation for the search of parameterizations capable of producing more accurate profiles.

A study performed a few years ago at the southern site of the Pierre Auger Observatory (Argentina), derived an improved model including also temperature, pressure and vapor pressure profiles [12]. In particular, the atmospheric depth profile is characterized by a layered structure similar to the approach made by Linsley. An important feature of this model is the fact that the experimental data, obtained from atmospheric balloons, are grouped in monthly bins giving a set of 12 parameterizations with different layer boundaries, one for each calendar month (thus called Malargüe Monthly Model). With this model, the experimental data can be reproduced with remarkable accuracy. Considered as a parameterization, the monthly model satisfies the premise of being a fit function that represents the data precisely, however it introduces an artificial temporal discontinuity in the atmospheric depth profile, so that the profile provided by the model for an air shower event in the last hour of the last day of a month is different than the one provided for an event occurring an hour later.

The Malargüe Monthly Model (MMM) and its update New Malargüe Monthly Model (NMMM) [1] have been used in the atmospheric characterization of simulation, detection and reconstruction of cosmic rays events at the Pierre Auger Observatory. Both models were developed with atmospheric measurements from a database made in the Pierre Auger Observatory ${ }^{[1]}$.

\section{THE GAMMA MODEL}

The starting point of this work is the interest in a characterization of the atmospheric profile by physical parameters instead of temporal considerations.

Among all the physical quantities that could affect the atmosphere, the temperature is one of the most influential for the density profile. Considering this, the temperature at ground appears as a good parameter to characterize the atmospheric depth profiles. On this basis, we introduce the GAMMA (Ground temperature Association to Multilayer isothermal Model of the Atmosphere) model. In our model, the vertical depth profile has the same functional form as equation (4), that

[1] The MMM also includes measurements from balloons launched at Santa Rosa and Cordoba, two cities located approximately at $500 \mathrm{~km}$ and $650 \mathrm{~km}$ from Malargüe. 
is,

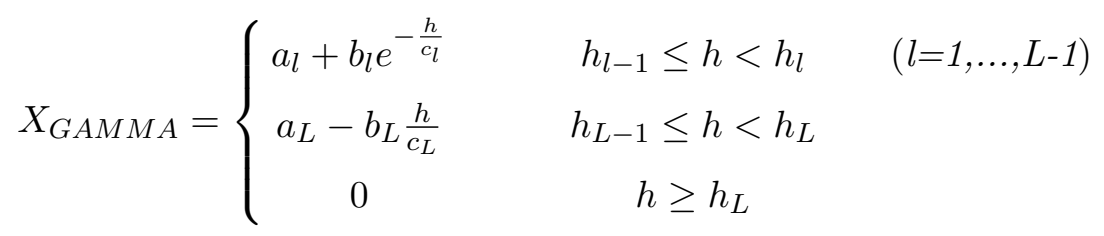

where $L$ is the total number of layers, $h_{L}=h_{\text {lim }}$ is the maximum altitude (top of the atmosphere), and the coefficients $a_{l}, b_{l}, c_{l}, l=1, \ldots, L$ are now functions of the temperature to be adjusted conveniently, as they are discussed in the following sections.

\section{A. Procedure}

1. The Database.

The atmospheric measurements used in our work were obtained from weather balloons launched at the Pierre Auger Observatory. In order to establish the conditions in which the measurements were made, it is important to recall that the Pierre Auger Observatory [13], capable of studying cosmic rays with energy above $10^{17} \mathrm{eV}$, has a southern site located near the city of Malargüe, in Mendoza (Argentina), approximately at $35^{\circ} 14^{\prime} \mathrm{S}-69^{\circ} 15^{\prime} \mathrm{W}$, at a plateau with average altitude of 1420 m.a.s.l. The weather is dry with low precipitations $\left(280 \mathrm{~mm} \mathrm{year}^{-1}\right)$ and with a mean annual temperature of $12{ }^{\circ} \mathrm{C}$ (Mean Minimum $-2{ }^{\circ} \mathrm{C}$ - Mean Maximum $20^{\circ} \mathrm{C}$ ). In addition to other devices to monitor the atmospheric status, there is a Balloon Launching Station (BLS) with intermittent launches of weather balloons at the array of the Pierre Auger Observatory .

The atmospheric measurements from weather balloons used in this study have been performed in periodical campaigns; and later in a more regular manner, generally in about every 5 days. The balloons were launched throughout the year at different hours of the day and night trying to produce a representative sample of different atmospheric conditions. Some groups of launches were performed within a small interval of few hours, allowing to observe fast variations in the parameters at low altitude. With these measurements, we made a database composed by 277 balloons launches that represent 277 atmospheric profiles between August 2002 and December 2008.

The meteorological balloons carried radiosondes capable of taking data every 8 seconds [14]. This ensures altitude dependent profiles of temperature $T(h)$, air pressure $p(h)$ and relative humidity $u(h)$ with high resolution [15]. The parameters needed to calculate the atmospheric depth profile can be obtained from these profiles. In particular, the air density $\rho(h)$ can be calculated, 
using the ideal gas law, as

$$
\rho(h)=\frac{p(h) M_{m}(h)}{R T(h)}
$$

where $R$ is the universal gas constant and $M_{m}(h)$ is the air molar mass expressed in $\mathrm{g} \mathrm{mol}^{-1}$. The molar mass includes the water vapor contribution extracted from the humidity profile [16], so that its value deviates slightly from the constant $M_{m}$ previously defined.

The atmospheric depth profile can be obtained integrating the density from the top of the atmosphere down to the ground level. For a series of estimations of the density $\rho\left(h_{i}\right)$ at given altitudes $h_{i}$, where the index $i$ labels the different measurements within the same balloon ascent, the atmospheric depth profile can be estimated by means of the following approximation

$$
\begin{aligned}
X\left(h_{i}\right) & =X\left(h_{i+1}\right)+\Delta X \\
\Delta X & \approx \frac{\rho\left(h_{i}\right)+\rho\left(h_{i+1}\right)}{2} \cdot\left(h_{i+1}-h_{i}\right) .
\end{aligned}
$$

The starting point used in our calculations corresponds to the maximum height reached by the atmospheric balloon, that is, the altitude of balloon burst $h_{b}$. The initial value for the series defined in equation $(7), X\left(h_{b}\right)$, comes from the approximation

$$
X\left(h_{b}\right) \approx \frac{p\left(h_{b}\right)}{g\left(h_{b}\right)}
$$

where

$$
p\left(h_{b}\right)=\int_{h_{b}}^{\infty} g(h) \cdot \rho(h) d h \approx g\left(h_{b}\right) \int_{h_{b}}^{\infty} \rho(h) d h
$$

and $g(h)$ is the gravity acceleration at altitude $h$.

In order to check the consistency of the different data sets, we have analyzed two additional quantities that can be obtained from the measured atmospheric profiles, namely, the quotient between the pressure versus height and atmospheric depth, and the quotient between pressure and density multiplied by temperature versus height. The analytical expressions of these quantities are

$$
C(h)=\frac{p(h)}{\rho(h) T(h)} \quad \text { and } \quad G(h)=\frac{p(h)}{X(h)}
$$

respectively.

For an ideal gas, the first expression corresponds to the ratio between the gas constant $R$ and the molar mass, that is truly independent of altitude in the case of dry air ( $M_{m}$ as already defined). The second should give the approximate dependence of the gravity with altitude. It is found that 
in most measurements $G(h)$ and $C(h)$ behave as expected within a typical range of small deviations that are characteristic of every set of experimental measurements: the profile $G(h)$ has a linear behavior and the $C(h)$ profile diminishes slightly from $288.0 \mathrm{~m}^{2} \mathrm{~s}^{-2} \mathrm{~K}^{-1}$ at ground to $287.1 \mathrm{~m}^{2}$ $\mathrm{s}^{-2} \mathrm{~K}^{-1}$ at approximately $5 \mathrm{~km}$ of height, and remains constant above this limit.

However, in a small subset of 33 measured profiles, the resulting $G(h)$ and/or $C(h)$ deviate significantly from the corresponding expectations ${ }^{[2]}$. The results of our analyses are not significantly affected by the elimination of these problematic 33 profiles. For this reason and to improve the robustness of our study, we have decided to exclude that set of measurements.

We have grouped the data set in ten bins of temperatures according to the value of the temperature at ground, covering a range between $278 \mathrm{~K}$ and $298 \mathrm{~K}$ (Table II). These values of temperature at ground have been obtained from a linear fit of the temperature profile in the first $5.6 \mathrm{~km}$. We find it worthwhile mentioning here that, as a posteriori test, we have compared the ground temperatures obtained via the mentioned method with the corresponding ground temperatures recorded by the Pierre Auger Observatory weather stations (when available), finding no significant differences between them. We have also studied possible effects of inversion in temperature, which can be an usual phenomenon in arid climate. Very few cases with a slight inversion were detected (about $2 \%$ ), but considering the small fraction have not been discarded.

In summary, we take into account 212 profiles for this work, discarding 32 profiles with temperature at ground lower than $278 \mathrm{~K}$ or higher than $298 \mathrm{~K}$ which would imply to consider bins with 16 low statistics. For each bin we built the profile in altitude of the atmospheric depth average.

\begin{tabular}{|c|c|c|}
\hline Bin & T range $[\mathbf{K}]$ & Measured Profiles \\
\hline 1 & $278-280$ & 15 \\
\hline 2 & $280-282$ & 17 \\
\hline 3 & $282-284$ & 20 \\
\hline 4 & $284-286$ & 26 \\
\hline 5 & $286-288$ & 23 \\
\hline 6 & $288-290$ & 25 \\
\hline 7 & $290-292$ & 22 \\
\hline 8 & $292-294$ & 27 \\
\hline 9 & $294-296$ & 19 \\
\hline 10 & $296-298$ & 18 \\
\hline
\end{tabular}

[2] We cannot give a closed explanation of the possible reasons that could originate these deviations, due fundamentally to the lack of information about the experimental details and the environmental conditions during the problematic measurements. 
TABLE II: Distribution of the profiles in ground temperature bins.

\section{The fit.}

We performed a series of analysis to determine the function that allows to adjust precisely the vertical depth profiles provided by the experimental data.

From these analyses we concluded that $L=7$ layers is the most appropriate number of layers to use in equation (5). We also determined the layers boundaries that provide accurate fits with the average profiles of each temperature bin.

The selected layer limits can be found in Table III.

\begin{tabular}{|c|c|c|c|c|c|c|c|}
\hline Layer & $\mathbf{1}$ & $\mathbf{2}$ & $\mathbf{3}$ & $\mathbf{4}$ & $\mathbf{5}$ & $\mathbf{6}$ & $\mathbf{7}$ \\
\hline Range $[\mathrm{km}]$ & $0-3.5$ & $3.5-8.5$ & $8.5-16$ & $16-24$ & $24-40$ & $40-100$ & $100-112.8$ \\
\hline
\end{tabular}

TABLE III: Layers limits of GAMMA Model

The average maximum height reached by the balloons is around $26 \mathrm{~km}$, a typical maximum altitude for such devices. Nevertheless, there is no special need for taking data at higher altitudes since in this region the USStdA 1976 values are accurate enough for our case. For this reason, we decided to adjust the vertical depth profile using the five lowest layers (that correspond to altitudes located in the region reached by the balloons, see Table III) in appropriate fits to the available data, and set the remaining coefficients so that $X_{G A M M A}(h)$ matches Linsley's profile for all altitudes corresponding to layers 6 and 7 . This also implies that the coefficients $a_{l}, b_{l}$, and $c_{l}$, $l=6,7$ of equation (5) are temperature independent.

The fits to the different data sets have been performed imposing the constraint of continuity between consecutive layers for $X_{G A M M A}(h)$ and its first derivative, including continuity at the top of layer 5 with Linsley's profile and its derivative at the corresponding altitude. No constraints were imposed at ground level.

With those considerations, the number of free parameters to be adjusted in the fit reduces to 5 , that can conveniently be chosen $c_{1}, c_{2}, c_{3}, c_{4}, c_{5}$. The remaining coefficients $a_{l}$ and $b_{l}$, $l=1,2,3,4,5$, can be evaluated using a straightforward procedure described in the Appendix. 

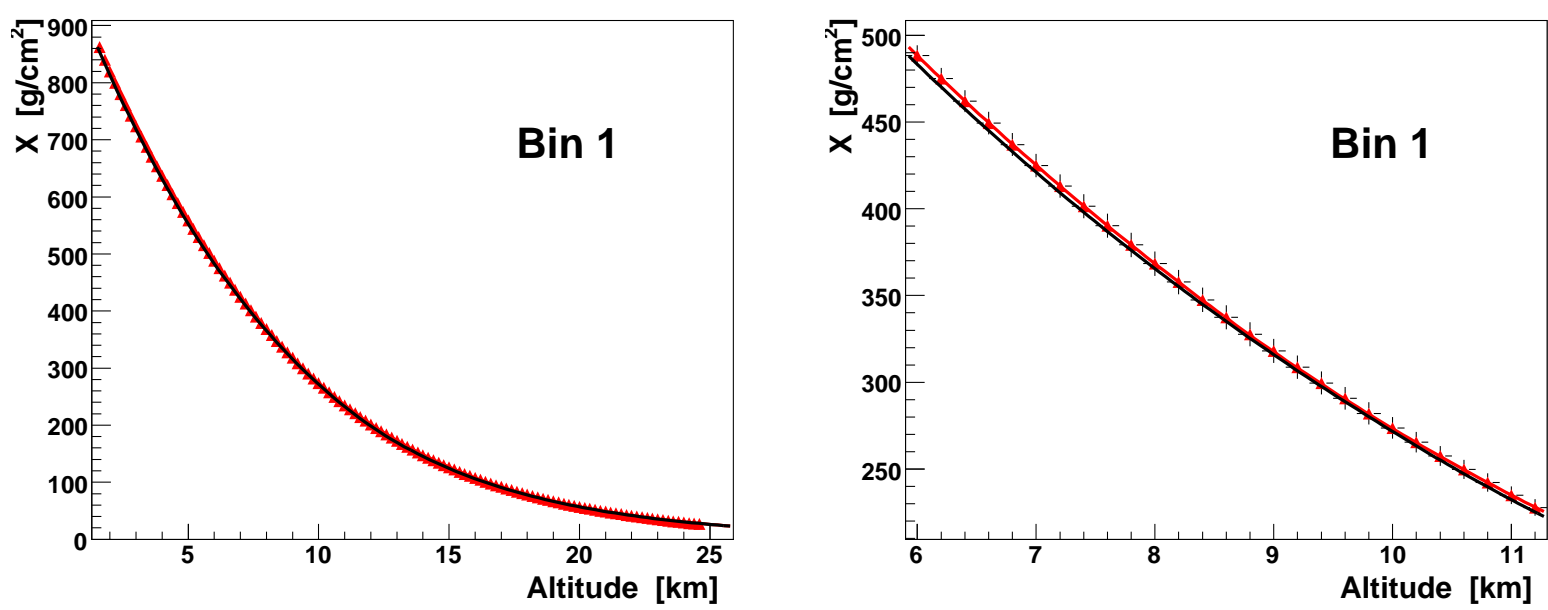

FIG. 1: Average atmospheric depth versus altitude, corresponding to Bin 1 . The red line is the parameterization of $X(h)$. Full range plot (left) and zoom (right) to appreciate the precision of the fit, practically coincident with the averaged data points. The black line corresponds to the Linsley Model of 5 layers, which underestimates slightly the averaged data points.

\section{B. Outcome}

The proposed parameterization reproduces adequately the experimental data (Figures 1 (left) and 2 (left)). The parameterization matches the data more precisely than the Linsley Model and this is more evident as the temperature at ground increases (Figure 1 (right) and more significantly in Figure 2 (right)).

From the fit we obtain the parameters $c_{l}, l=1,2,3,4,5$, for each temperature bin. We have plotted the values of $c_{l}$ to analyze its temperature dependence (Figure 3). As a first approximation a linear fit was done, and the parameters obtained for these linear fits are shown in Table IV. A first look over the coefficients $c_{1}$ and $c_{2}$ in Figure 3, leads to think that a linear function does not fit the data appropriately. Using a third degree polynomial results in a significantly better fit. However, such better fit does not result in substantial improvements in the overall matching of $X_{\text {GAMMA }}$ with the data. For that reason we decided to keep linear functions to describe the temperature dependence of all the coefficients $c_{l}, l=1,2,3,4,5$.

The results from the linear fits define entirely the GAMMA Model: using the ground level temperature, it is now possible to calculate univocally the corresponding atmospheric depth profile using equation (5) with the corresponding layer limits (Table III).

The values of the parameters $c_{l}(l=1, \ldots, 5)$ are obtained using the expression 

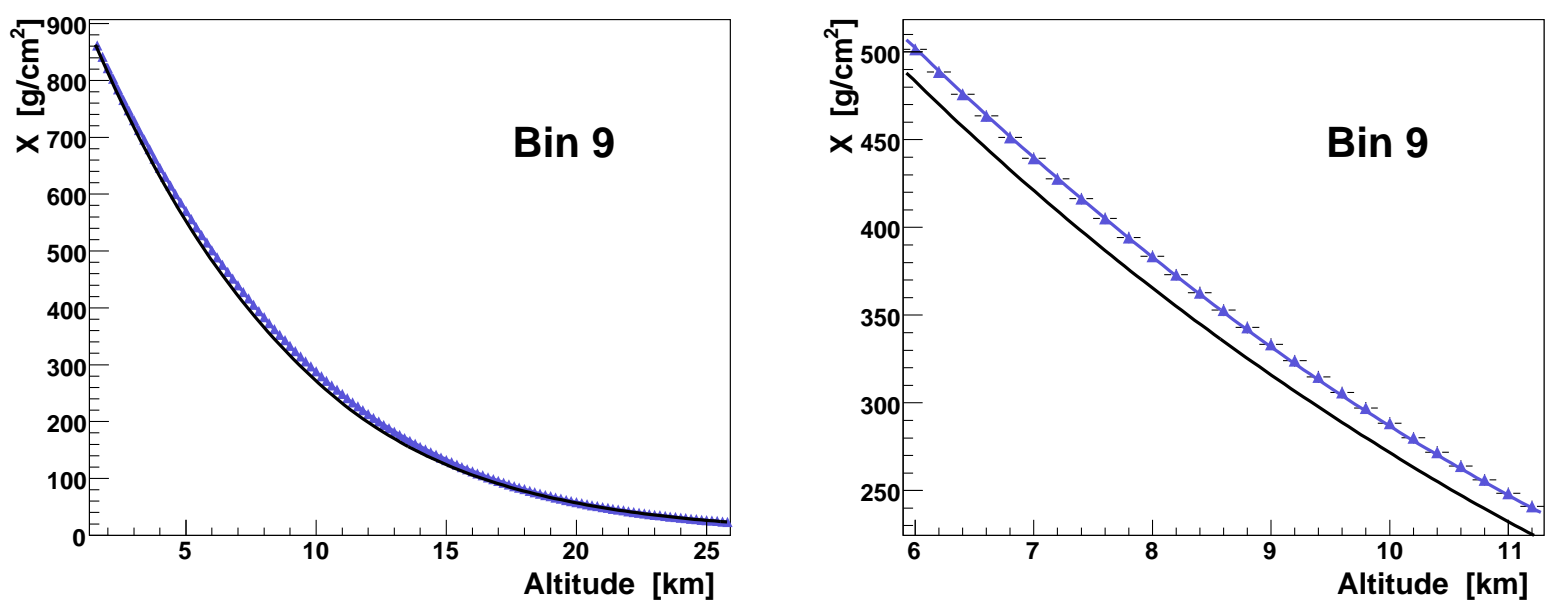

FIG. 2: Average atmospheric depth versus altitude, corresponding to Bin 9. The blue line is the parameterization of $X(h)$. Full range plot (left) and zoom (right) to appreciate the precision of the fit, practically coincident with the averaged data points. The black line corresponds to the Linsley Model of 5 layers, which as very well known underestimates the averaged data points.

\begin{tabular}{|c|c|c|}
\hline Layer (l) & $N_{l}[\mathrm{~km}]$ & $M_{l}\left[\mathrm{~km} \mathrm{~K}^{-1}\right]$ \\
\hline $\mathbf{1}$ & -20.716 & 0.1034 \\
$\mathbf{2}$ & -0.626 & 0.0376 \\
$\mathbf{3}$ & -1.64 & 0.0307 \\
$\mathbf{4}$ & 9.668 & -0.0129 \\
$\mathbf{5}$ & 7.463 & -0.00038 \\
\hline
\end{tabular}

TABLE IV: Values of the parameterization $c_{l}\left(T_{G R O U N D}\right)=M_{l} T_{G R O U N D}+N_{l}$.

$$
c_{l}\left(T_{G R O U N D}\right)=M_{l} T_{G R O U N D}+N_{l} \quad(l=1, \ldots, 5)
$$

where $\mathrm{M}_{l}$ and $\mathrm{N}_{l}$ are listed in Table IV. The values of $a_{l}$ and $b_{l}, l=1, \ldots, 5$, can be derived from the expressions given in the Appendix. Finally, the values of $a_{l}, b_{l}$ and $c_{l}, l=6,7$, correspond to the Linsley model parameterization and can also be found in the Appendix.

It is important to mention that the temperature interval $\left[T_{\min }, T_{\max }\right]=[278 \mathrm{~K}, 298 \mathrm{~K}]$ that corresponds to the experimental data can be used to define a first range of validity of the model in the present case.

In order to analyze the precision achieved by the parameterization, we took the value of the temperature at ground for each experimental profile $X_{E X P}(h)$ and we calculated the atmospheric 

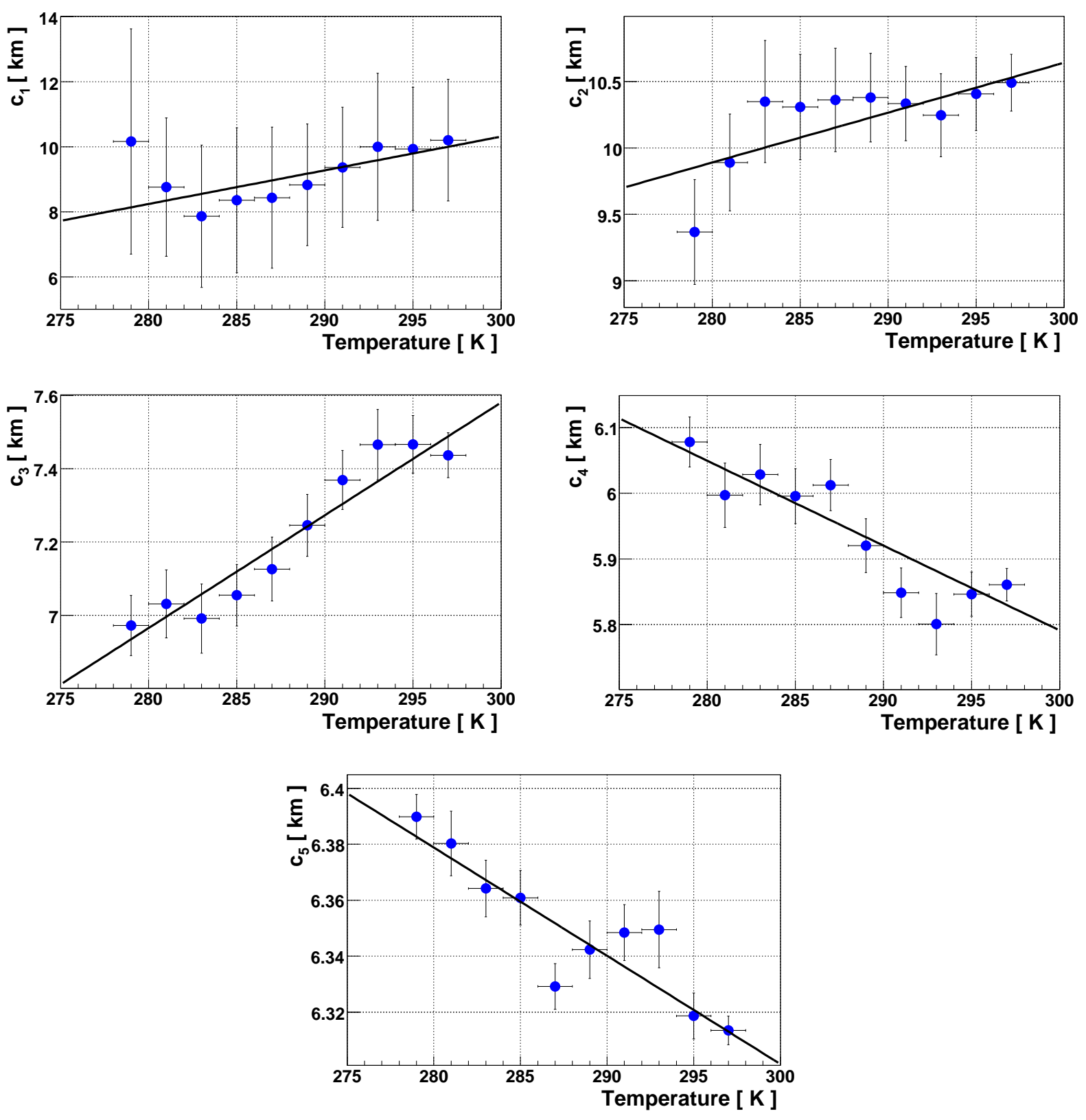

FIG. 3: $c_{1}, c_{2}, c_{3}, c_{4}, c_{5}$ versus temperature. The dots correspond to the different temperature bins. Fitted linear functions are also shown; the corresponding fit parameters are listed in Table IV.

profile $X_{\text {GAMMA }}(h)$ predicted by the model at those temperatures. Defining the residual as

$$
\operatorname{Residual}(h)=X_{E X P}(h)-X_{G A M M A}(h)
$$

we made some plots to analyze the behavior of the residuals in each bin (Figures 4 and 5). It can be seen that the differences between the GAMMA Model and the experimental data in each 
bin are smaller than approximately $2 \mathrm{~g} \mathrm{~cm}^{-2} \pm 4 \mathrm{~g} \mathrm{~cm}^{-2}$ (approximately $0.7 \% \pm 1.4 \%$ ).
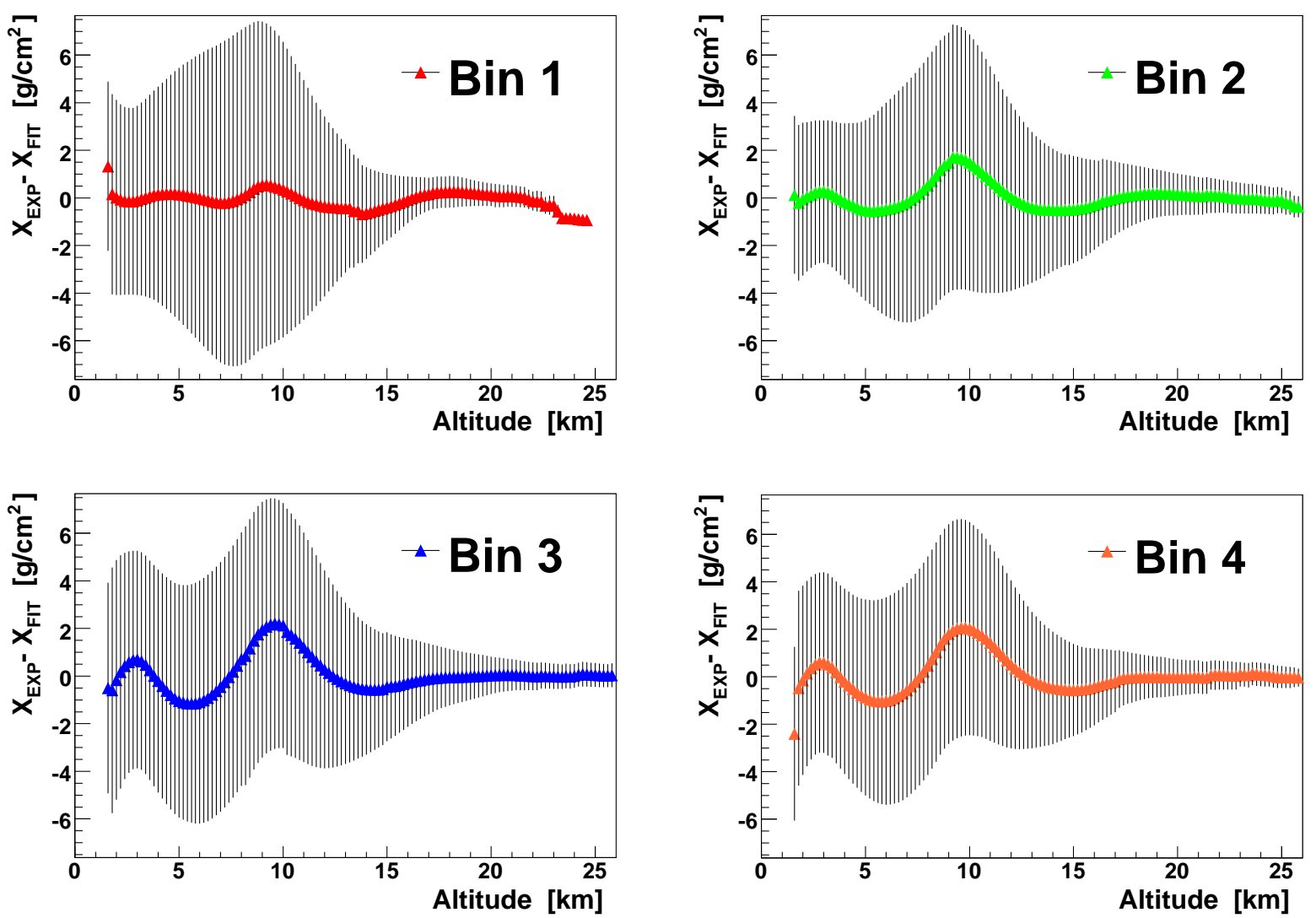

FIG. 4: GAMMA Model average residuals versus altitude (Bins 1, 2, 3 and 4).

If we take all the experimental data without discrimination in bins and we plot the average residuals versus altitude, we obtain that the average uncertainty predicted by the GAMMA Model is smaller than approximately $2 \mathrm{~g} \mathrm{~cm}^{-2} \pm 4 \mathrm{~g} \mathrm{~cm}^{-2}$ (Figure 6 a). Similarly, the experimental data and the prediction of the New Malargüe Monthly Model of the corresponding month are compared (Figure $6 \mathrm{~b}$ ). The average uncertainty is consistent with that achieved by the GAMMA Model. As it was expected, there are greater differences between the prediction by the Linsley Model and the experimental data (Figure $6 \mathrm{c}$ ).

With the aim of checking the validity of the GAMMA Model at the Malargüe site, we have also calculated the residuals over a new independent set of data (43 profiles collected in the period January 2009 - August 2010) (Figure 6 d). The maximum values observed in the average residuals are approximately $3 \mathrm{~g} \mathrm{~cm}^{-2} \pm 4 \mathrm{~g} \mathrm{~cm}^{-2}$, slightly larger than in the case of the original data. 

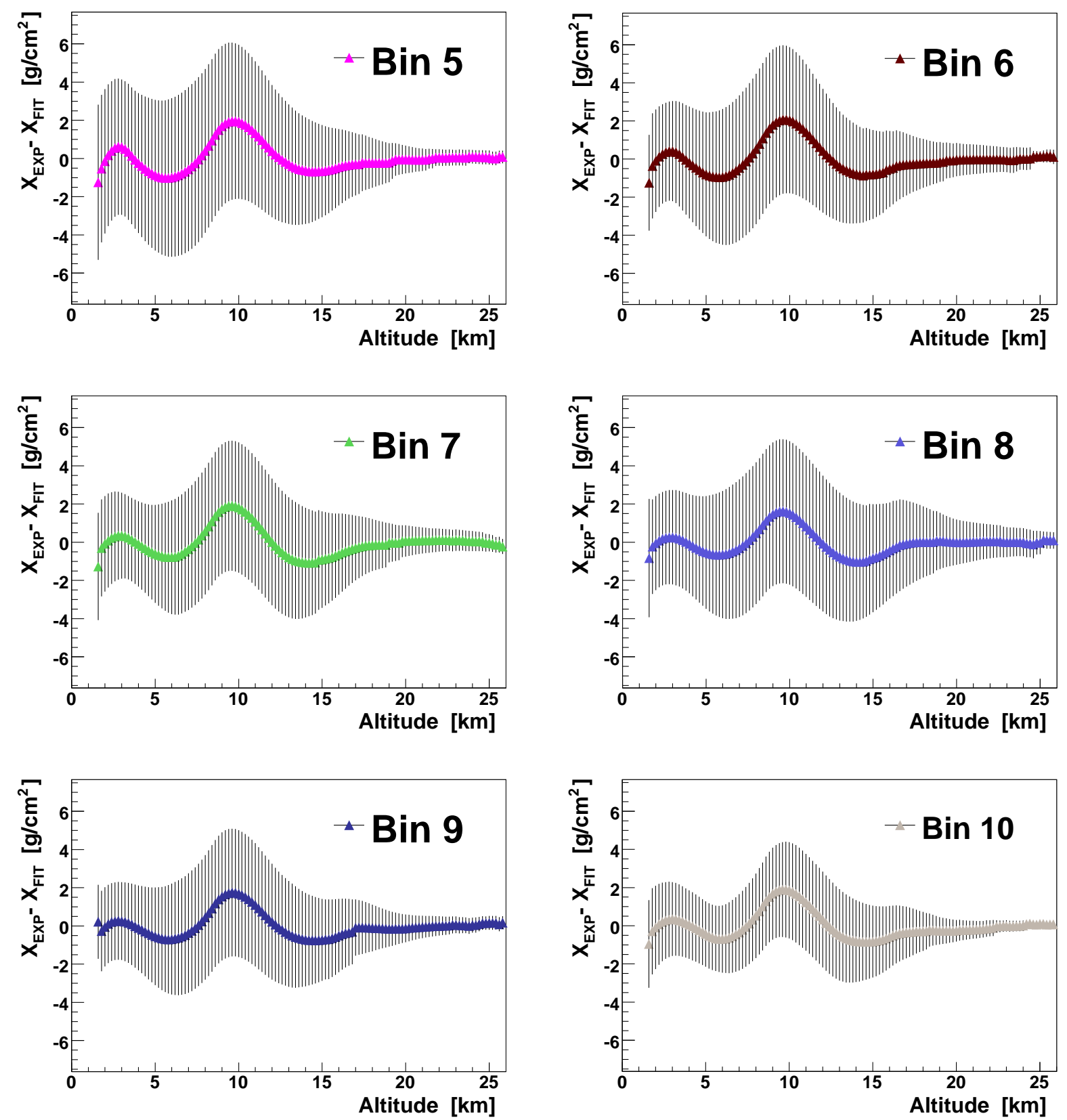

FIG. 5: GAMMA Model average residuals versus altitude (Bins 5, 6, 7, 8, 9 and 10).

\section{DISCUSSION}

Several points of the obtained results need special analysis and discussion.

Firstly, it is important to note that the comparisons between GAMMA and NMMM (Figures $6(\mathrm{a})$ and $6(\mathrm{~b})$ ) correspond to average values, but in the analysis of particular profiles, the differences between the GAMMA and the NMMM could be different. A particular situation could happen 


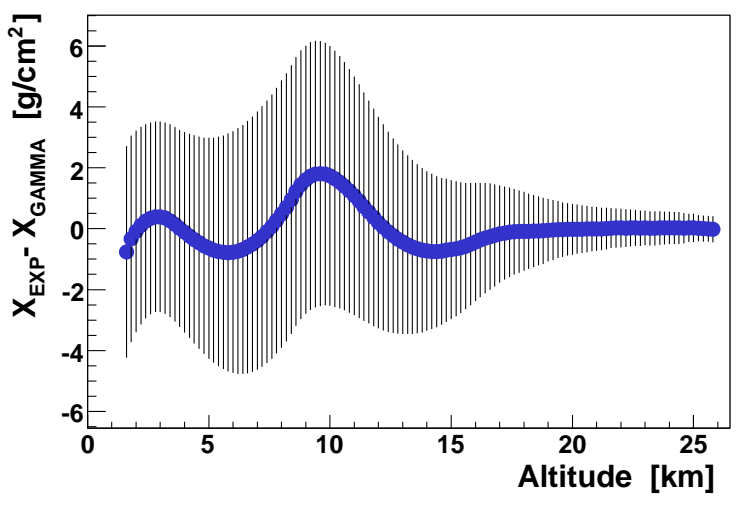

(a) GAMMA Model - Original database.

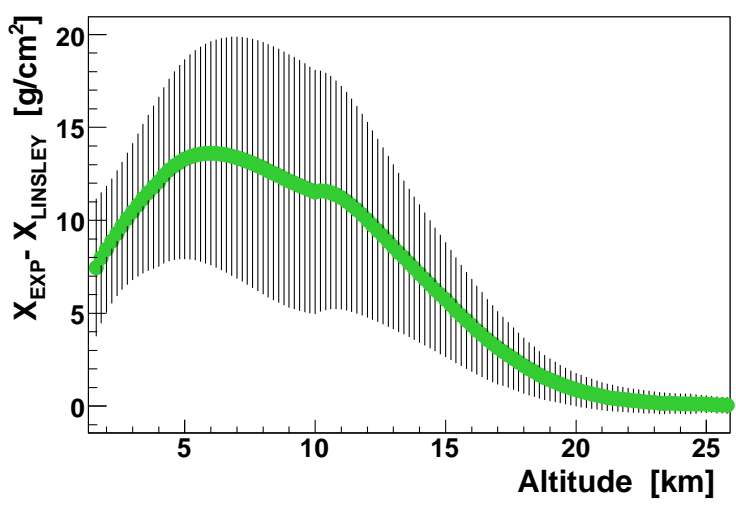

(c) Linsley Model.

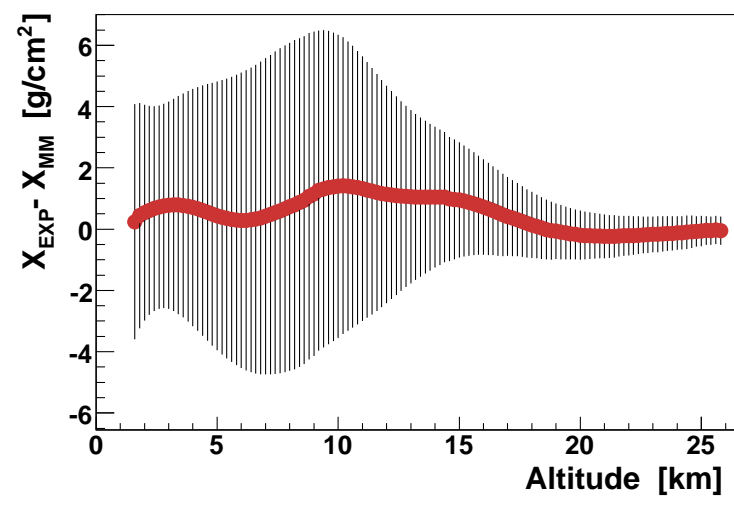

(b) New Malargüe Monthly Model.

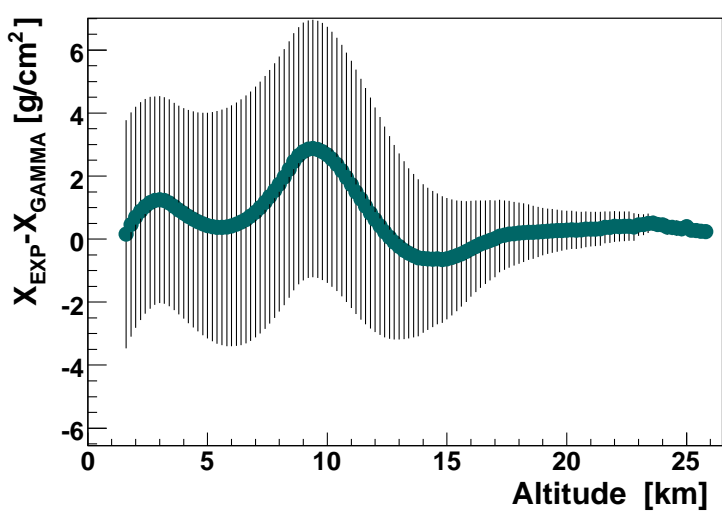

(d) GAMMA Model - New database.

FIG. 6: Average residuals versus altitude including all the data: GAMMA Model (a), New Malargüe Monthly Model (b), Linsley Model (c), and check of the GAMMA Model using a new independent database (d).

with those profiles whose associated ground temperature is substantially larger or smaller than the corresponding monthly average. In such cases, it could happen that the predictions of the NMMM (invariant during the whole month) and the GAMMA (depending upon temperature) models differ more than in normal cases. We have found within the experimental data available some profiles that are examples of this situation. In these cases, the differences between the GAMMA Model and these particular profiles are similar to those reported in this work, that is, approximately $2 \mathrm{~g} \mathrm{~cm}^{-2}$ $\pm 4 \mathrm{~g} \mathrm{~cm}^{-2}$, while the NMMM model presents for the same cases differences of up to more than $7 \mathrm{~g}$ $\mathrm{cm}^{-2}$. This fact demonstrates an important feature of our model: while the NMMM profiles remain constant within a given month, the GAMMA Model ones are sensitive to the changes induced by daily ground temperature variations.

In the comparison between the GAMMA and Linsley models (Figures 1, 2, 6 (a) and 6 (c)), the first one reproduces the atmospheric depth profiles substantially better. We must mention that 
the improved performance of the GAMMA Model derives from the fact that the $X_{G A M M A}$ profile comes from fits to local atmosphere measurements. Even though the GAMMA and Linsley models are based on different databases, the comparison between these models is still relevant because the latter is a global model built upon data collected at many geographical locations distributed around the world [9]; what makes it an usual option when local data are not available. We have thus compared the predicted profiles by both models, considering several values of temperature at ground. This study allows to observe that the average difference between the models increases with ground temperature (Figure 7), reaching at the highest temperatures considered in this work, values that are significantly larger than the residuals between the GAMMA Model and the corresponding measured profiles (that are practically independent of temperature, as clearly illustrated in figures 4 and 5). Beyond that we are comparing with a model built with data from Malargüe, this promotes two readings: (i) a warning signal in the use of the Linsley Model, especially at high temperatures; and (ii) the validation of the temperature at ground as a good parameter to characterize the atmospheric depth profiles.

From the analysis performed with the independent database (Figure $6 \mathrm{~d}$ ), we conclude that the small difference observed in the average residuals is a consistency test passed by the GAMMA Model. We observe that the structure of the average residuals in Figure 6 (a) and Figure 6 (d) is very similar. We estimate that this behavior could be a consequence of the number of layers and its limits.

The GAMMA Model temperature range is another point to be discussed. Originally, the model was developed using profiles corresponding to temperatures at ground level in the range $278 \mathrm{~K}$ $298 \mathrm{~K}$. We have compared the predictions of the GAMMA Model for the parameters presented here with profiles corresponding to ground temperatures out of the mentioned range (19 profiles between the limits $[269.9 \mathrm{~K}, 278 \mathrm{~K}]$ and 13 profiles in the range $[298 \mathrm{~K}, 304 \mathrm{~K}]$ that were originally not taken into account in the model set up, see section III A 1). From this analysis, we could observe that the residuals corresponding to the profiles with ground temperatures greater than $298 \mathrm{~K}$, show a similar behavior than the ones analyzed in section III B. However, this is not observed at temperatures below $278 \mathrm{~K}$ where the residuals become significantly larger. Both cases are illustrated at Figure 8. From this analysis, we can conclude that the GAMMA Model can be extended to the temperature range from $278 \mathrm{~K}$ to $304 \mathrm{~K}$.

When using the GAMMA Model to reproduce the depth profile, we suggest that an appropriate choice is to employ the average temperature evaluated over a few hours interval. This is a valid selection since the fluctuations in the temperature at ground within such a period, has no immediate 


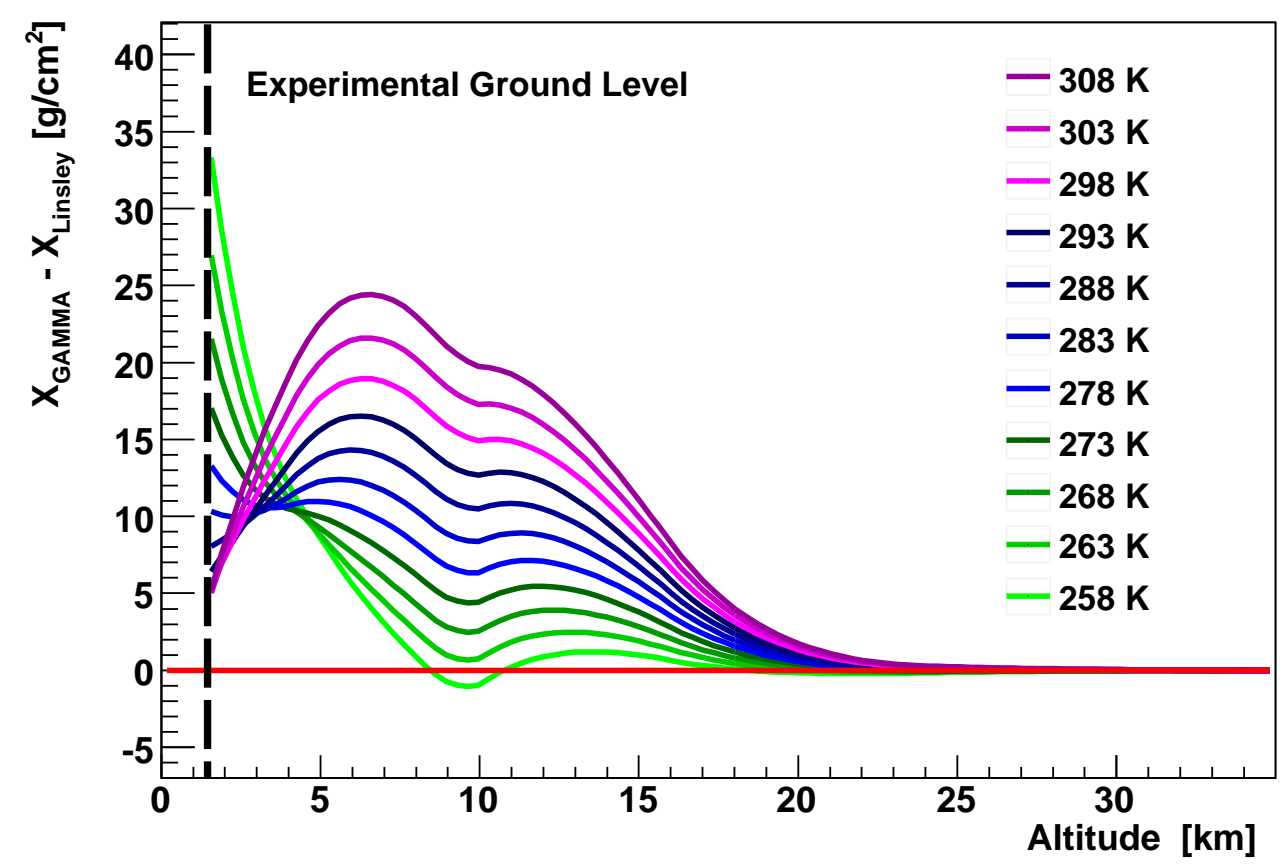

FIG. 7: Differences between the profile predicted by the Linsley Model of 5 layers and several profiles obtained from the GAMMA Model with diverse temperatures at ground, plotted as a function of altitude. In order to observe the trend beyond the range defined for GAMMA, the temperature range explored was extended including the cases $308 \mathrm{~K}, 273 \mathrm{~K}, 268 \mathrm{~K}, 263 \mathrm{~K}$ and $258 \mathrm{~K}$. The dashed line indicates the ground level corresponding to the experimental database.
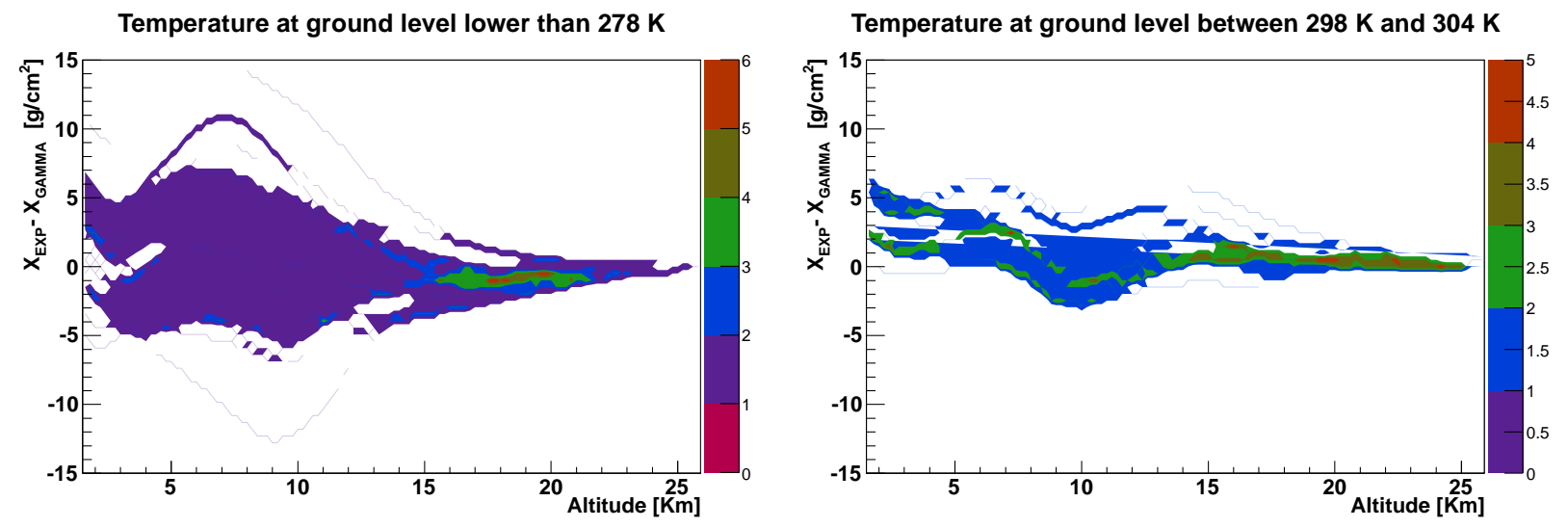

FIG. 8: Difference between the GAMMA Model prediction and the experimental profiles with extreme ground temperatures, as a function of altitude: 19 profiles with temperature lower than $278 \mathrm{~K}$ (left) and 13 profiles with temperature higher than $298 \mathrm{~K}$ (right). 
influence on the complete atmospheric depth profile.

The GAMMA Model could be improved including more parameters, besides the temperature at ground, to characterize the atmospheric depth profile. The parameters to be included should be measured preferably at ground level so they can be easily obtained, thus preserving the spirit of the model. The pressure at ground (combined with the temperature at ground) is among the bests candidates being investigated to be included in an updated version of our model.

The results we have presented in our analysis are based on data corresponding to the Malargüe site as a study case. Nevertheless, the GAMMA Model could be implemented to obtain the atmospheric depth profiles from other locations, for example sites corresponding with other cosmic ray experiments. Work is in progress in this direction.

\section{SUMMARY AND CONCLUSIONS}

A new model for atmospheric depth profile $X(h)$, the GAMMA Model, has been presented. We have built the model driven by two motivations: (i) the accurate description of the local atmosphere, improving the well-known Linsley Model that uses the USStdA database; and (ii) the need to take into account the atmospheric profiles variations as a function of a physical parameter (alternatively to NMMM that uses a temporal one). With these premises, the model includes the assumption that the temperature at ground is a suitable indicator for the atmospheric conditions at any moment. The database used comes from radiosondes that were launched at the southern site of the Pierre Auger Observatory and the function proposed in the model keeps the layers structure of the Linsley Model.

The parameterization obtained describes with an excellent agreement the experimental data. Particularly, the value of the atmospheric depth at ground given by the GAMMA parameterization has a significant accuracy, being the average difference with data less than $0.9 \mathrm{~g} \mathrm{~cm}^{-2} \pm 3 \mathrm{~g} \mathrm{~cm}^{-2}$ (approximately $0.11 \% \pm 0.36 \%$ ). These low percentages are remarkable considering that the fit was made with free boundary conditions at ground level. The high accuracy at ground level could be appreciated in Figures 4, 5 and 6 (a).

Considering the entire profiles, the average difference between the data and the model are smaller than approximately $2 \mathrm{~g} \mathrm{~cm}^{-2} \pm 4 \mathrm{~g} \mathrm{~cm}^{-2}$. These results are of the same order than the ones corresponding to the New Malargüe Monthly Model. In percentages, the differences are smaller than $0.7 \%( \pm 1.4 \%)$ in all bins (Figures 4 and 5$)$. The average residuals profile made over the complete data presents the same differences (Figure 6 (a)) and it has also been shown that the 
model could be extended to $304 \mathrm{~K}$ keeping the uncertainty values already mentioned.

\section{APPENDIX}

The function $X_{\text {GAMMA }}$ defined by the GAMMA Model uses the parameters $a_{l}, b_{l}$ and $c_{l}$ $(l=1, . ., 5)$. The parameters $c_{l}$ have to be calculated in first place, using in equation (12) the temperature at ground level and the values of $M_{l}$ and $N_{l}$ (Table IV).

The parameters $a_{l}$ and $b_{l}$ remain univocally defined by the imposition of boundary conditions and the continuity between layers of $X_{G A M M A}(h)$ and its derivative, as discussed in section III A 2 . These conditions can be expressed as

$$
\begin{gathered}
b_{5}=0.000381472 c_{5} e^{-h_{5} / c_{5}} \mathrm{~g} \mathrm{~cm}^{-2} \\
b_{4}=b_{5} \frac{c_{4}}{c_{5}} e^{-h_{4} / c_{5}} e^{h_{4} / c_{4}} \mathrm{~g} \mathrm{~cm}^{-2} \\
b_{3}=b_{4} \frac{c_{3}}{c_{4}} e^{-h_{3} / c_{4}} e^{h_{3} / c_{3}} \mathrm{~g} \mathrm{~cm}^{-2} \\
b_{2}=b_{3} \frac{c_{2}}{c_{3}} e^{-h_{2} / c_{3}} e^{h_{2} / c_{2}} \mathrm{~g} \mathrm{~cm}^{-2} \\
b_{1}=b_{2} \frac{c_{1}}{c_{2}} e^{-h_{1} / c_{2}} e^{h_{1} / c_{1}} \mathrm{~g} \mathrm{~cm}^{-2} \\
a_{5}=\quad 3.0396-b_{5} e^{-h_{5} / c_{5}} \mathrm{~g} \mathrm{~cm}-2 \\
a_{4}=a_{5}+b_{5} e^{-h_{4} / c_{5}}-b_{4} e^{-h_{4} / c_{4}} \mathrm{~g} \mathrm{~cm}^{-2} \\
a_{3}=a_{4}+b_{4} e^{-h_{3} / c_{4}}-b_{3} e^{-h_{3} / c_{3}} \mathrm{~g} \mathrm{~cm}^{-2} \\
a_{2}=a_{3}+b_{3} e^{-h_{2} / c_{3}}-b_{2} e^{-h_{2} / c_{2}} \mathrm{~g} \mathrm{~cm}^{-2} \\
a_{1}=a_{2}+b_{2} e^{-h_{1} / c_{2}}-b_{1} e^{-h_{1} / c_{1}} \mathrm{~g} \mathrm{~cm}^{-2}
\end{gathered}
$$

The values of $a_{l}, b_{l}$ and $c_{l}(l=6,7)$ are derived from the Linsley Model of 5 layers:

$$
\begin{array}{ll}
a_{6} & =0 \quad 0 \quad \mathrm{~g} \mathrm{~cm}^{-2} \\
b_{6} & =540.1778 \mathrm{~g} \mathrm{~cm}^{-2} \\
c_{6} & =7721.7016 \mathrm{~m} \\
a_{7} & =0.01128292 \mathrm{~g} \mathrm{~cm}^{-2} \\
b_{7} & =1.0 \quad \mathrm{~g} \mathrm{~cm}^{-2} \\
c_{7} & =10000000 \mathrm{~m}
\end{array}
$$

\section{ACKNOWLEDGMENTS}

This study was enriched with comments of the Atmospheric Monitoring group of the Pierre Auger Observatory. We are also deeply indebted to the crew of the Balloon Launching Station 
that measured the data used in this work. One of the authors (JCM) acknowledges the valuable interest and commentaries of the Pierre Auger Observatory group from University of Tor Vergata (Rome, Italy). We are grateful to the members of the Pierre Auger Observatory group from La Plata, particularly to M. Tueros and A. Mariazzi, and we are indebted to C. A. García Canal for useful discussions.

This work was partially supported by Agencia Nacional de Promoción Científica y Tecnológica and the Consejo Nacional de Investigaciones Científicas y Técnicas (Argentina). Also thanks to the High Energy Latin american-European Network.

\section{REFERENCES}

[1] J. Abraham et al., The Pierre Auger Collaboration, A study of the effect of molecular and aerosol conditions in the atmosphere on air fluorescence measurements at the Pierre Auger Observatory, Astropart. Phys. 33 (2010) 108-129.

[2] M. Sasano et al., HiRes Collaboration, Atmospheric monitoring in Utah using the Back Scatter Lidar, in: Proceedings of the 27th International Cosmic Ray Conference (Hamburg, Germany), 2001, 653-656.

[3] K. M. Aye et al., HESS Collaboration, Atmospheric monitoring for the HESS project, in: Proceedings of the 28th International Cosmic Ray Conference (Tsukuba, Japan), 2003, 2879-2882.

[4] C.M. Hui for the VERITAS Collaboration, VERITAS Distant Laser Calibration and Atmospheric Monitoring, in: AIP Conf.Proc. 1085: 735-737, 2009.

[5] T. Yamamoto et al., The Telescope Array Collaboration, Telescope array atmospheric monitoring system at Akeno Observatory, in: Proceedings of the 27th International Cosmic Ray Conference (Hamburg, Germany), 2001, 663-666.

[6] B. Wilczyǹska, D. Góra, P. Homola, B. Keilhauer, H. Klages, J. Pekala and H. Wilcyzs̀kin, Importance of Atmospheric Model in Shower Reconstruction, in: Proceedings of the 28th International Cosmic Ray Conference (Tsukuba, Japan), 2003, 571-574.

[7] S. Sciutto, The AIRES system for air shower simulations: An Update, in: Proceedings of the 27th International Cosmic Ray Conference (Hamburg, Germany), 2001. See also AIRES User's Manual and Reference Guide version 2.6.0 (2002), available electronically at www.fisica.unlp.edu.ar/auger/aires.

[8] D. Heck and T. Pierog, CORSIKA User's Guide version CORSIKA 6.7 (2007), available electronically at www-ik.fzk.de/corsika/usersguide/corsika_tech.html.

[9] National Aeronautics and Space Administration (NASA), US-Standard Atmosphere 1976, NASA-TMX-74335 (1976), http://modelweb.gsfc.nasa.gov/atmos/us_standard.html.

[10] We were not able to find official references related with Linsleys standard atmosphere model. The References A. M. Hillas, Shower simulations: Lessons from MOCCA ,Nucl. Phys. B (Proc. Suppl.), 
52B, 29 (1997); and A. M. Hillas, in: Proceedings of the 19th International Cosmic Ray Conferenc (La Jolla, USA), 1, 155 (1985), contain information about parameterization data.

[11] B. Wilczyǹska, D. Góra, P. Homola, J. Pekala, M. Risse and H. Wilcyzs̀kin, Variation of atmospheric depth profile on different time scales, Astropart. Phys. 25 (2006) 106-117.

[12] J. Blümer, R. Engel, D. Gora, P. Homola, B. Keilhauer, H. Klages, J. Pekala, M. Risse, M. Unger, B. Wilczynska, H. Wilczynski for the Pierre Auger Collaboration, Atmospheric Profiles at the Southern Pierre Auger Observatory and their Relevance to Air Shower Measurement, in: Proceedings of the 29th International Cosmic Ray Conference (Pune, India), 2005, 7, 123.

[13] The Pierre Auger Observatory Design Report, www.auger.org.

[14] B. Keilhauer, J. Blümer, R. Engel, H.O. Klages, M. Risse, Impact of Varying Atmospheric Profiles on Extensive air shower Observation: - Atmospheric Density and Primary Mass Reconstruction, Astropart. Phys. 22 (2004) 249-261.

[15] For the performance of the radiosondes used to acquire the atmospheric data at the Malargüe Pierre Auger Observatory, see: www.graw.de (model GRAW DFM-97).

[16] B. A. Bodhaine, N. B. Wood, E. G. Dutton, and J. R. Slusser, On Rayleigh optical depth calculations, Jou. Atmos. Ocean. Tech., (1999), 16, 1854-1861. 